\title{
Determinación de los niveles de plomo en sangre en trabajadores de fábricas de baterías ubicadas en Guayaquil-Ecuador
}

\section{(Determination of lead levels in blood in workers of battery factories located in Guayaquil-Ecuador)}

\author{
Kathya Rivera' ${ }^{1}$ Beatriz Pernía ${ }^{2}$
}

\begin{abstract}
Resumen
El plomo es uno de los metales pesados más tóxicos y la exposición humana, por razones laborales, a este metal, puede causar plumbemia. El objetivo de la investigación fue generar una línea base sobre las concentraciones de plomo en sangre de un grupo de trabajadores de fábricas de baterías en Guayaquil-Ecuador y de un grupo control no expuesto laboralmente al metal, con el fin de establecer la existencia de riesgo ocupacional entre sexos, edades y puestos de trabajo. Para ello, se procedió a tomar muestras de sangre a los trabajadores y al grupo control. Las muestras fueron analizadas por espectrofotometría de absorción atómica con horno de grafito. El 100 \% de los trabajadores presentaron Pb en una concentración promedio de 16.22 $26.82 \mu \mathrm{g} / \mathrm{dL}$ superior al valor de la Organización Mundial de la Salud, pero inferior al límite establecido por la Administración de Seguridad y Salud Ocupacional de $40 \mu \mathrm{g} / \mathrm{dL}$; además, el valor fue superior al del grupo control $(0.68 \pm 0.15 \mu \mathrm{g} / \mathrm{dL})$. Los hombres presentaron mayores niveles de $\mathrm{Pb}$ que las mujeres, y estos valores variaron dependiendo del puesto de trabajo en la fábrica. En conclusión, el 100 \% del grupo control y el $4.55 \%$ de los trabajadores no presentaron riesgo de intoxicación por plomo, un 13.64 \% presentó riesgo bajo, $78.79 \%$ riesgo medio y solo un $3.00 \%$ riesgo alto.
\end{abstract}

\section{Palabras clave}

Contaminación, plomo, fábricas de batería, riesgo ocupacional.

\begin{abstract}
Lead is one of the most toxic heavy metals and its occupational exposure can generate plumbemia. For this reason, the objective of this investigation was to generate a baseline of lead concentrations in the blood of a group of battery factory workers in Guayaquil-Ecuador and of a control group not exposed to the metal at work, to establish the existence of occupational risk between sexes, ages, and jobs positions. For this, blood samples were taken from the workers and the control group. The samples were analyzed by atomic absorption spectrophotometry with a graphite furnace. $100 \%$ of the workers presented Pb in an average concentration of $16.22 \pm 6.82 \mu \mathrm{g} / \mathrm{dL}$ higher than the World Health Organization value, but lower than the limit established by Occupational Health and Safety Assessment of 40 $\mu \mathrm{g} / \mathrm{dL}$, also, the value was higher than of the control group $(0.68 \pm 0.15 \mu \mathrm{g} / \mathrm{dL})$. Men presented higher levels of $P b$ than women and these values varied depending on the job in the factory. In conclusion, $4.55 \%$ of the workers did not present a risk of lead poisoning, $13.64 \%$ low risk, $78.79 \%$ medium risk, and only $3.00 \%$ high risk.
\end{abstract}

\section{Keywords}

Contamination, lead, battery factories, occupational risk.

$1 \quad$ Facultad de Ciencias Naturales. Universidad de Guayaquil, Guayaquil-Ecuador [kathyavanina@gmail.com, https://orcid. org/0000-0002-5272-6036]

2 Instituto de Investigaciones de Recursos Naturales. Universidad de Guayaquil, Guayaquil-Ecuador [beatriz.pernias@ug.edu. ec, https://orcid.org/0000-0002-2476-7279] 


\section{Introducción}

El plomo (Pb) es un metal que los seres humanos han utilizado desde hace más de 9000 años. Sin embargo, debido a su naturaleza no biodegradable y su uso generalizado, se ha convertido en un contaminante omnipresente (Devóz et al., 2017; Himani et al., 2020). Este se ha usado para elaborar baterías, balas, escudos de radiación, joyas de plata, soldaduras, remedios caseros, aditivos de gasolina, tuberías de distribución de agua, esmaltes de cerámica y se ha reconocido como un contaminante ambiental y ocupacional ( Agency for Toxic Substances and Disease Registry [ATSDR], 2020; Ballantyne et al., 2018). Además, el plomo se encuentra en los entornos urbanos y, en especial, en zonas residenciales cercanas a las industrias (Dean et al., 2020; Fry et al., 2020).

Las personas adultas están expuestas principalmente al plomo en sus lugares de trabajo, ya sea a través de la inhalación de partículas cargadas de plomo, la falta de higiene personal o el consumo de agua y alimentos contaminados (Basit et al., 2015; Stojsavljevi et al., 2019). Tras la exposición, el plomo se absorbe fácilmente en el tracto gastrointestinal y se transporta rápidamente a la sangre y a los tejidos blandos con una vida media de 28-36 días y luego se acumula en los huesos con una vida media de 27 años (ATSDR, 2020).

Una vez que el $\mathrm{Pb}$ ingresa en el organismo, causa numerosos efectos adversos para la salud, incluidos daños en los riñones y en los sistemas reproductivo, nervioso, cardiovascular y hematopoyético (Chen et al., 2019; Gottesfeld \& Pokhrel, 2011). También ocasiona disrupciones endócrinas, genotoxicidad, inhibición de la actividad enzimática, muerte celular y problemas inflamatorios (Devóz et al., 2017). La Agencia Internacional para la Investigación del Cáncer ha clasificado al plomo y a los compuestos de plomo dentro del Grupo 2A de probables carcinógenos humanos (Nersesyan et al., 2016). Sin embargo, según Steenland y Boffetta (2000) la Agencia Internacional para la Investigación del Cáncer clasificó el plomo en el año 1987 como un "posible carcinógeno humano" basándose en que existían suficientes datos sobre su efecto en los animales, pero insuficientes datos en humanos. En la actualidad, hay numerosas investigaciones que demuestran la correlación entre los niveles de plomo y el cáncer.

En este sentido, Ahn et al. (2020) realizaron un metaanálisis donde analizaron 222 publicaciones y concluyeron que existe evidencia de asociación entre el plomo en sangre y la incidencia de tumores cerebrales, y observaron un mayor riesgo ante la exposición laboral a este metal. De igual manera, Steenland y Boffetta (2000) y Steenland et al. (2019) demostraron asociación entre los niveles de plomo en sangre con cáncer de pulmón y de cerebro (Chowdhury et al., 2014), y Meng et al. (2020) determinaron que el plomo puede ser un factor de riesgo de meningiomas y cáncer de cerebro. También se ha encontrado correlación entre el contenido de plomo y cáncer de laringe (Barry y Steenland, 2019), de estómago (Rousseau et al., 2007), de vejiga (Awadalla et al., 2020) y de mama en mujeres (Alatise y Schrauzer, 2010).

En adición, en los niños, la concentración de plomo en la sangre se asocia con una disminución significativa en el rendimiento académico y puntuaciones más bajas en las pruebas de coeficiente intelectual y está relacionado con el comportamiento hiperactivo y violento (Gaza et al., 2017). Este metal se elimina del cuerpo de forma lenta, principalmente a través de la orina, las heces, el cabello, las uñas y el sudor, y por ello se va acumulando con los años (Yabe et al., 2018).

La industria de las baterías es la mayor consumidora de plomo y se estima que aproximadamente el 80 \% de la producción mundial de plomo se utiliza para fabricar las baterías, y aproximadamente el 50 \% de esa producción se deriva del reciclaje de las mismas baterías 
(Gottesfeld \& Pokhrel, 2011). La fabricación de baterías de plomo implica preparar óxido de plomo, fundición de rejillas, pegado, corte de placas, formación, carga y ensamblaje; y, para ello, se utilizan productos químicos peligrosos tales como óxido de plomo, plomo esponjoso y ácido sulfúrico (Kalahasthi et al., 2019).

Esta industria se está expandiendo rápidamente en países con mercados emergentes y aumenta por el incremento en la demanda de vehículos. Se estima que, entre 60000 y 70000 personas están empleadas a nivel mundial en la fabricación de baterías de plomo, y la gran mayoría de estos trabajadores vive en países en vía de desarrollo, donde las medidas de control de metales pesados no son tan estrictas (Nouioui et al., 2019). Es por ello que para monitorear el contenido de plomo en estos trabajadores se utiliza como marcador biológico predominante la determinación de los niveles de plomo en sangre, que es utilizado en la evaluación clínica, el control del lugar de trabajo, la vigilancia de la salud pública y las decisiones de las autoridades reguladoras (Baloch et al., 2020; Stojsavljevi et al., 2019).

En este contexto, a nivel mundial se han descrito niveles promedio de plomo en sangre de trabajadores de fábrica de baterías de $47 \mu \mathrm{g} / \mathrm{dL}$ en las plantas de fabricación de baterías y $64.00 \mu \mathrm{g} / \mathrm{dL}$ en las instalaciones de reciclaje (Gottesfeld \& Pokhrel, 2011). De acuerdo con el Centro de Control y Prevención de Enfermedades de Estados Unidos, el valor a partir del cual se consideran niveles elevados de Pb en todos los grupos de la población es $5 \mu \mathrm{g} / \mathrm{dL}$; según la OMS, el límite máximo permisible es $10 \mu \mathrm{g} / \mathrm{dL}$ (Téllez-Rojo et al., 2017). En el caso de exposición laboral, la Conferencia Americana de Industria Gubernamental propone un límite máximo de $30 \mu \mathrm{g} /$ dL y la Administración de Seguridad y Salud Ocupacional indica que el límite de tolerancia en personas adultas expuesta a Pb es de $40 \mu \mathrm{g} / \mathrm{dL}$. Con base en estos valores y a la revisión hecha por Gottesfeld \& Pokhrel (2011) en trabajadores de fábricas de batería a nivel mundial, se evidencia que el personal presenta altos niveles de $\mathrm{Pb}$, los cuales pueden ser peligrosos para su salud.

En Ecuador, se ha reportado que el $46 \%$ de los trabajadores de una fábrica de batería en Quito presentaron niveles altos de plomo en sangre (Cevallos, 2013). En la investigación de Cevallos (2013) se encontraron niveles de plomo entre 5.40 a $79.50 \mu \mathrm{g} / \mathrm{dL}$ Pb. Así mismo, Cruz (2015) reportó en otra fábrica de baterías, en Quito, que el 71 \% de la población analizada presentaba niveles altos de plomo en sangre de 3.30 a $79.90 \mu \mathrm{g} / \mathrm{dL}$. Sin embargo, se desconoce el nivel de plomo de los empleados de las fábricas de baterías de Guayaquil.

Guayaquil es la segunda ciudad más poblada de Ecuador, después de Quito, con una población de 2,7 millones de habitantes y es el principal centro económico, de recursos culturales y financieros de Ecuador (Instituto Nacional de Estadísticas y Censos, 2015). En esta ciudad se hallan numerosas industrias dentro de las que se encuentran fábricas de baterías.

El objetivo de la investigación fue generar una línea base de concentraciones de plomo en sangre de trabajadores de fábricas de baterías en Guayaquil-Ecuador y de personas no expuestas laboralmente al metal; comparar los niveles de Pb según el sexo, edad, años de exposición y puestos de trabajo en la empresa para analizar los posibles riesgos asociados, y proponer algunas recomendaciones para mitigar los riesgos de exposición al Pb. La hipótesis planteada fue que las personas expuestas laboralmente al plomo muestran mayores concentraciones de plomo en sangre que las no expuestas y presentan un mayor riesgo de sufrir intoxicación por plomo. 


\section{Metodología}

\section{Área de estudio}

Ecuador se encuentra en Suramérica, en la zona ecuatorial y en las costas del Océano Pacífico (Torres Del salto et al., 2017). La segunda ciudad más poblada y la más importante a nivel comercial de este país es Guayaquil, ubicada en la provincia de Guayas, en la Costa ecuatoriana, situada en la zona de vida bosque muy seco tropical (Figura 1). Guayaquil está ubicada en la margen occidental del río Guayas (79 $50^{\prime}-79^{\circ} 59^{\prime} \mathrm{W}$ y $\left.2^{\circ} 02^{\prime}-2^{\circ} 18^{\prime} \mathrm{S}\right)$. Es la ciudad más grande del país, con 1800 km² de área total y una población estimada de 2,69 millones. El clima de Guayaquil es tropical húmedo, con una temperatura media anual de $26{ }^{\circ} \mathrm{C}$ y una precipitación media de 1030 mm (Johansson et al., 2018).

Figura 1. Área de estudio en el cantón Guayaquil
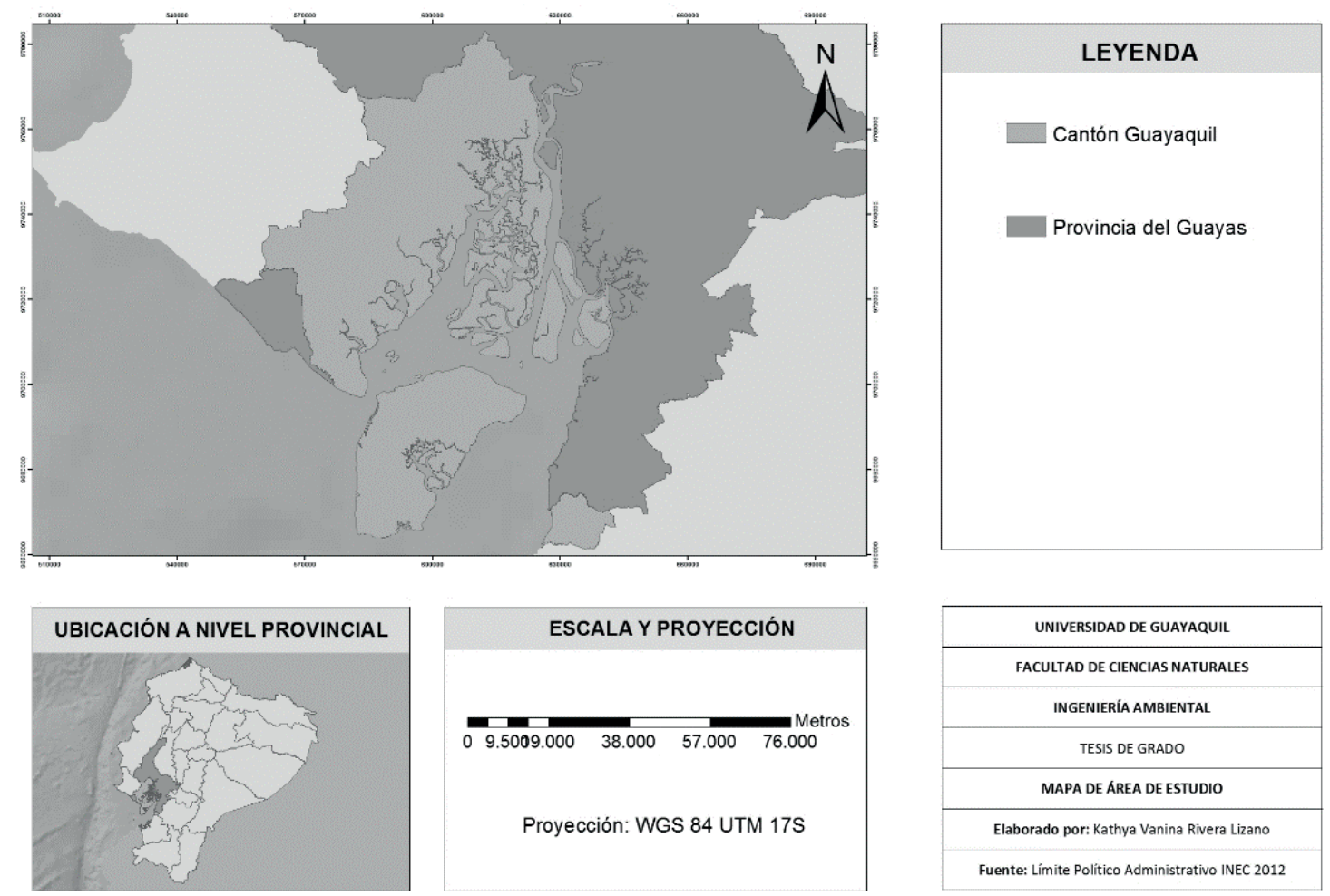

\section{Sujetos de estudio}

La presente investigación de línea base fue de tipo exploratoria, con muestreo discrecional. Para elegir a los sujetos, se utilizó como criterio de inclusión que los trabajadores tuvieran dos o más años laborando en la empresa, y como criterio de exclusión que no fueran fumadores. Las muestras se tomaron en época laboral, con exposición mínima al plomo de un día. Se tomaron muestras de 198 trabajadores de 3 fábricas de baterías ubicadas en Guayaquil, con un rango 
de edad de entre los 17 a los 62 años; dichos trabajadores fueron divididos en grupos según sus áreas de trabajo (Tabla 1).

Tabla 1. Puestos de trabajo de los empleados de las fábricas de baterías

\begin{tabular}{|l|c|}
\hline \multicolumn{1}{|c|}{ Puesto de trabajo } & \multicolumn{1}{|c|}{$\begin{array}{c}\text { Número de trabajadores } \\
\text { por puesto de trabajo }\end{array}$} \\
\hline Bodega de reciclaje & 3 \\
\hline Bodega Materia Prima & 11 \\
\hline Control de Calidad & 16 \\
\hline Empastado mezcladora & 10 \\
\hline Formación de baterías & 46 \\
\hline Fundición & 11 \\
\hline Ingeniería de mantenimiento & 32 \\
\hline Montaje & 47 \\
\hline Oficina & 1 \\
\hline Recursos Humanos & 2 \\
\hline Seguridad Industrial & 4 \\
\hline Supervisión & 11 \\
\hline Vicepresidencia & 4 \\
\hline Total & 198 \\
\hline
\end{tabular}

Para la población control de trabajadores, el criterio de inclusión fue tener edades en el rango de 20 a 60 años. En cuanto a los criterios de exclusión, deben estar expuesto laboralmente al plomo y ser fumador. 21 voluntarios cumplieron con los criterios. La muestra se tomó en noviembre de 2019. Se siguió el protocolo de Helsinski y se obtuvo el consentimiento informado por escrito de cada uno de los sujetos antes de participar en el estudio.

\section{Análisis de laboratorio}

En un laboratorio acreditado con la norma ISO 17025 se tomaron $5 \mathrm{~mL}$ de sangre venosa de los trabajadores de las fábricas de batería y el grupo control. $2 \mathrm{~mL}$ de sangre se guardaron en viales con EDTA para estimar el nivel de plomo en sangre y se almacenaron a $-20{ }^{\circ} \mathrm{C}$ hasta su análisis. Se digirieron $2 \mathrm{~mL}$ de sangre completa con $2 \mathrm{~mL}$ de ácido nítrico $\left(\mathrm{HNO}_{3^{\prime}}\right.$ Merck) y $0.2 \mathrm{~mL}$ de peróxido de hidrógeno $\left(\mathrm{H}_{2} \mathrm{O}_{2}\right.$, Merck) en un microondas marca Sineo (MDS-15) a la potencia, temperatura y tiempo adecuados. Las muestras digeridas se enrasaron hasta $5 \mathrm{~mL}$ con agua triple destilada. Las muestras debidamente rotuladas, se leyeron en un espectrofotómetro Perkin Elmer, modelo AAnalyst 400, con horno de grafito HGA 900 y auto muestreador AS 800. Además, se digirió y analizó una concentración conocida de solución estándar de plomo para el control de calidad interno.

\section{Análisis estadísticos}

Los análisis estadísticos se hicieron con el programa R versión 4.0.2. Se determinó la normalidad de los datos con la prueba de Kolmogorov-Sminorv, y el test de Levene se utilizó para determinar la igualdad de las varianzas entre los grupos. Para evidenciar si los hombres y mu- 
jeres de la población presentaban mayores concentraciones de Pb, se llevó a cabo una prueba T-Student para varianzas desiguales $(p<0.05)$.

A fin de comparar los niveles de plomo por edades, tiempo de exposición o puestos de trabajo de las personas laboralmente expuestas, se realizó una ANOVA de una vía y test a posteriori de Tukey $(p<0.05)$.

\section{Resultados}

En el grupo perteneciente a trabajadores de las fábricas de baterías, el 100 \% presentó plomo en sangre (Figura 2). En el grupo control, solo el $62 \%$ de los voluntarios mostraron Pb en sangre. Además, el promedio de $\mathrm{Pb}$ en sangre de los trabajadores de las fábricas de batería fue de $16.22 \pm 6.82 \mu \mathrm{g} / \mathrm{dL}$ significativamente mayor al grupo control $(0.68 \pm 0.15 \mu \mathrm{g} / \mathrm{dL})$ según $\mathrm{T}$ de Student $(T=-30.68 ; P<0.001)$.

En cuanto al sexo, en el grupo de trabajadores de las fábricas, el sexo femenino presentó un menor nivel de $\mathrm{Pb}$ que el masculino, con diferencias estadísticamente significativas según $\mathrm{T}$ de Student $(T=-19.05 ; P<0.001)$. Las mujeres mostraron concentraciones de plomo en sangre de $2.10 \pm 2.34 \mu \mathrm{g} / \mathrm{dL}$ menores en comparación con los hombres $(15.99 \pm 7.16 \mu \mathrm{g} / \mathrm{dL})$. Asimismo, en los hombres se mostraron valores atípicos de 37.00 y $38.30 \mu \mathrm{g} / \mathrm{dL}$ (Figura 3).

En el grupo control también se comprobó que los hombres presentaron mayores valores de plomo que las mujeres $(T=-2.89 ; P=0.010)$. En el sexo masculino se observó una media de $1.10 \pm 0.56 \mu \mathrm{g} / \mathrm{dL}$ y en el femenino $0.38 \pm 0.58 \mu \mathrm{g} / \mathrm{dL}$ (Figura 3). Se encontraron valores máximos de $\mathrm{Pb}$ de $1.50 \mu \mathrm{g} / \mathrm{dL}$ en mujeres y $2.20 \mu \mathrm{g} / \mathrm{dL}$ en hombres, los que son menores a los límites máximos permisibles de $10 \mu \mathrm{g} / \mathrm{dL}$ y menores al valor de la CDC de $5 \mu \mathrm{g} / \mathrm{dL}$, lo que indica que las personas no expuestas laboralmente a plomo no están en riesgo de sufrir envenenamiento por plomo.

Figura 2. Porcentaje de personas con plomo en sangre no detectable y detectable en la fábrica de baterías y el grupo control no expuesto ocupacionalmente
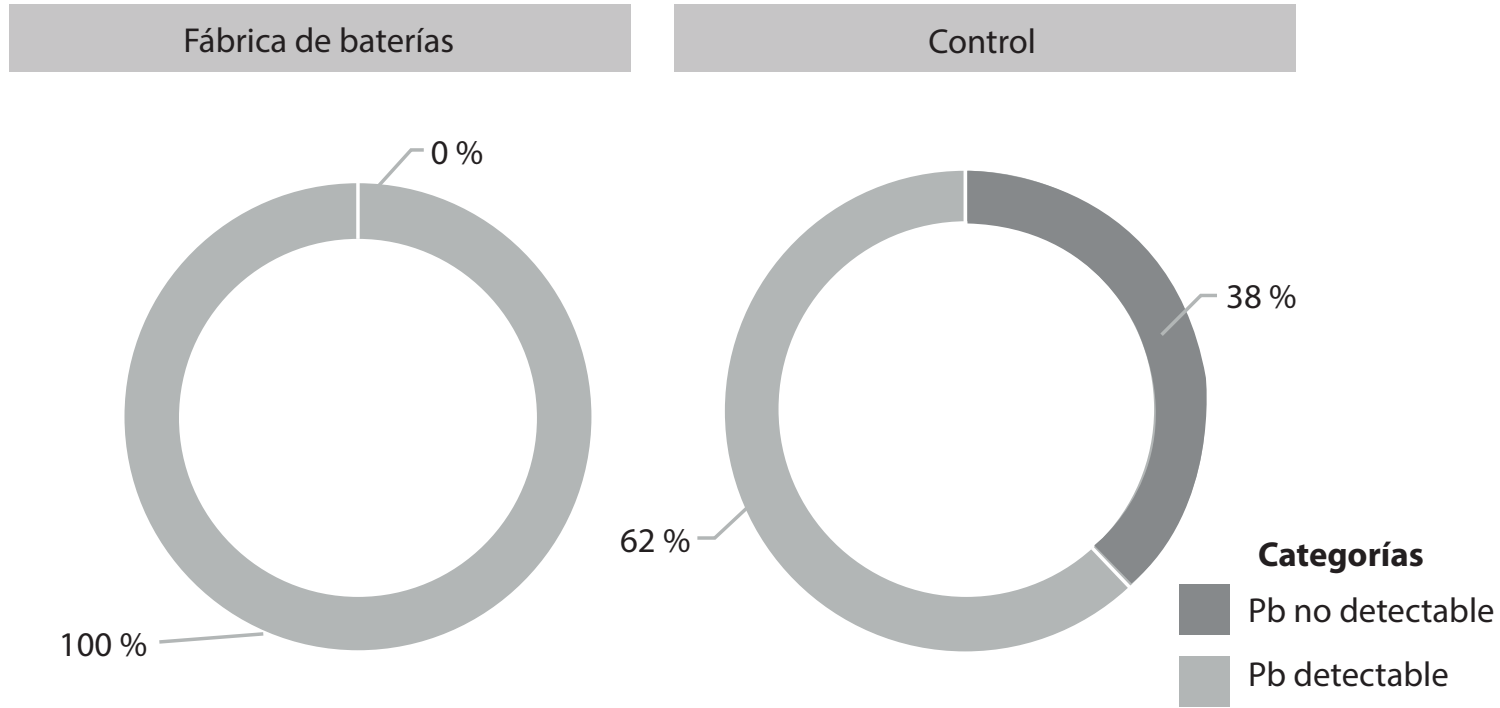
Figura 3. Concentración de plomo en sangre según el sexo en el grupo control y en los trabajadores de fábricas de baterías

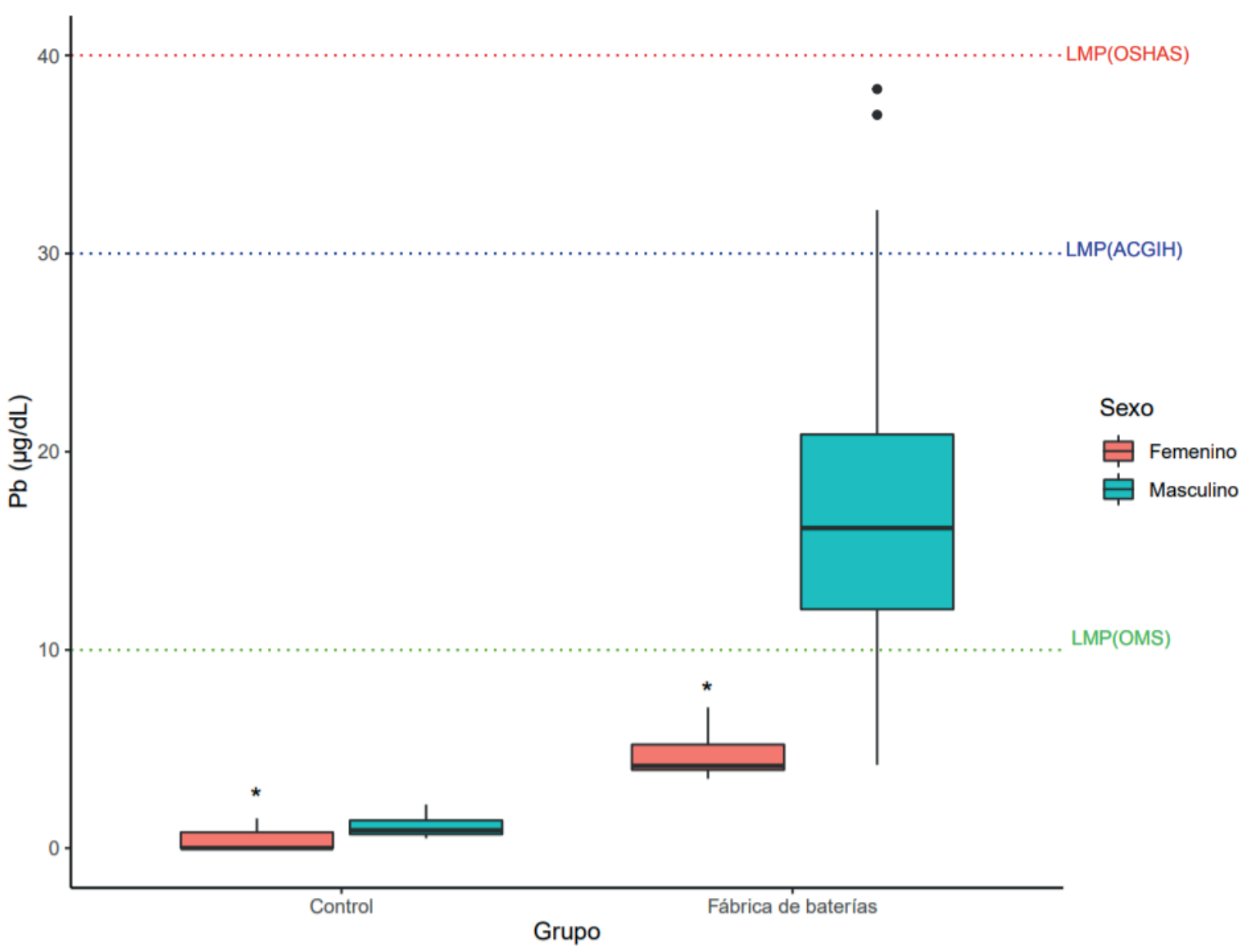

Nota: Línea verde: límite máximo permisible según la OMS (10 $\mu \mathrm{g} / \mathrm{dL})$; línea azul: límite máximo permisible según la American Conference Governmental Industrial Hygienists (ACGIH) es de $30 \mu \mathrm{g} / \mathrm{dL}$; línea roja: límite máximo permisible según la OSHAS es de $40 \mu \mathrm{g} /$ dL. El * indica que existen diferencias significativas según T de Student $(P>0.05)$.

Además del sexo, se comprobó que el puesto de trabajo es determinante en la contaminación por plomo en los trabajadores (Figura 4). Los niveles de Pb del personal de los diferentes puestos de trabajo se compararon con la población control, donde se observó que aquellos que trabajan en oficinas no presentaron diferencias significativas con las personas no expuestas, según la prueba de Tukey. Las personas del grupo control tuvieron un promedio de plomo en sangre de $0.69 \pm 0.15 \mu \mathrm{g} / \mathrm{dL}$, seguido por el personal de la fábrica de baterías que se encontraba en las oficinas con $3.80 \pm 0.00 \mu \mathrm{g} / \mathrm{dL}$; recursos humanos, $5.10 \pm 0.80 \mu \mathrm{g} / \mathrm{dL}$, y las que laboraban en seguridad industrial, $6.92 \pm 1.62 \mu \mathrm{g} / \mathrm{dL}$. Estos grupos se encontraron dentro de los límites máximos permisibles de la OMS (10 $\mu \mathrm{g} / \mathrm{dL})$.

Por el contrario, los individuos con elevadas concentraciones de $\mathrm{Pb}$ se encontraban en el área de montaje de baterías con un promedio de $21.00 \pm 0.97 \mu \mathrm{g} / \mathrm{dL}$, proceso de fundición, $20.33 \pm 1.33 \mu \mathrm{g} / \mathrm{dL}$; empastado mezcladora, $18.09 \pm 1.71 \mu \mathrm{g} / \mathrm{dL}$, y en el área de ingeniería de mantenimiento, $16.70 \pm 1.13 \mu \mathrm{g} / \mathrm{dL}$; además, se constataron diferencias significativas con el control 
( $F=15.73 ; P<0.001)$. Este grupo presentó un riesgo para su salud porque supera los límites máximos permisibles de la OMS, sin embargo, se encuentran dentro de los límites admisibles según la OSHA (10-40 $\mu \mathrm{g} / \mathrm{dL})$ para personas expuestas laboralmente al plomo.

Figura 4. Concentración de plomo en sangre de la población no expuesta al Pb (control) y en trabajadores de fábricas de baterías según su puesto de trabajo

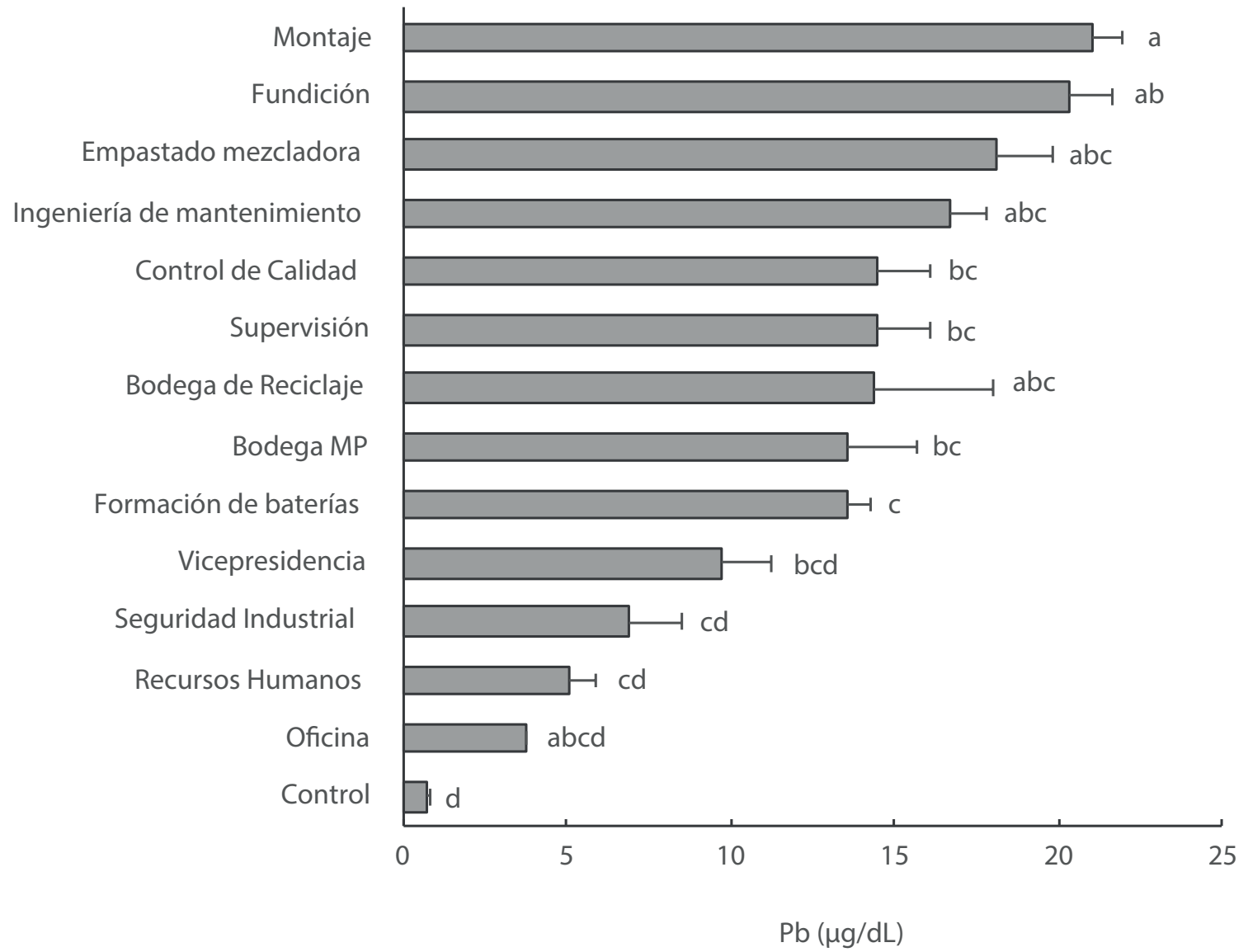

Nota: Los resultados se muestran como media đdesviación estándar. Letras iguales indican que no existen diferencias significativas según Anova de una vía y test a posteriori de Tukey $(P>0.05)$.

Con respecto a la edad del personal, en la Figura 5 se comprueba que no existe un patrón en la concentración del metal pesado por edades. La correlación lineal mostro un valor de $\mathrm{R}^{2}$ de 0.001, lo que indica que no existe correlación entre estas variables. De la misma manera, se aplicó un análisis de correlación de Pearson, que dio un valor de $r=-0.026$, por lo tanto, confirma que no existe correlación entre la edad y la concentración del metal pesado en sangre. 
Figura 5. Concentración de plomo en sangre según la edad (puntos) y sexo (rosado: femenino; azul: masculino)

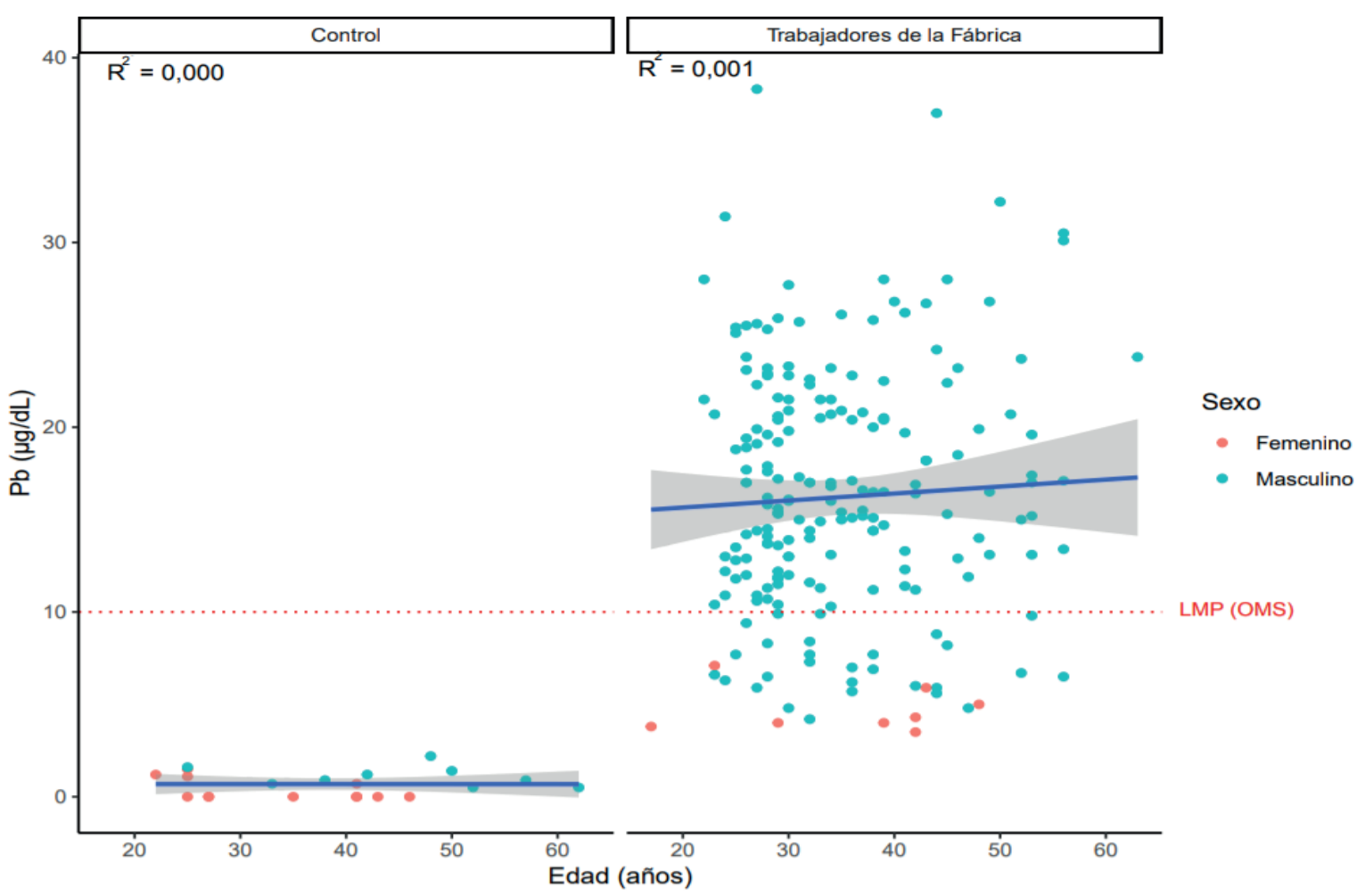

Nota: Línea azul: regresión lineal y línea roja: límite máximo permisible de plomo en sangre según la OMS

Los años de exposición al $\mathrm{Pb}$ tampoco fueron determinantes porque no se encontraron diferencias significativas entre la concentración de plomo en sangre con respecto a los años de trabajo en la fábrica de baterías $(P>0.05)$. Sin embargo, en la Figura 6 se observa que el promedio de plomo en sangre en los trabajadores con 2 años de exposición fue de $9.26 \pm 4.08 \mu \mathrm{g} /$ dL (min $=3.50$, máx $=13.00 \mu \mathrm{g} / \mathrm{dL} \mathrm{Pb}$ ) y los más antiguos en la compañía tenían 13 años de exposición y una concentración promedio de 16.79 $\pm 7.86 \mu \mathrm{g} / \mathrm{dL}$ ( $\min =4.30$, máx $=37.00 \mu \mathrm{g} / \mathrm{dL}$ ). También se apreció una correlación lineal moderada con un $\mathrm{R}^{2}$ de 0.48 entre la concentración del metal y los años de exposición (Figura 6).

En la Figura 7 se aprecia un análisis de componentes principales donde se observó que cuatro de los componentes explicaron el $93 \%$ de la correlación entre las variables. El primer componente (Dim1) mostró la correlación entre plomo en sangre con el cargo y el sexo, y el segundo componente (Dim2) una correlación entre los años de exposición y la edad de los trabajadores, lo que indica que existe mayor asociación entre altos niveles de $\mathrm{Pb}$ con el cargo y el sexo. 
Figura 6. Concentración de plomo en sangre por años de exposición en los trabajadores de las fábricas de baterías

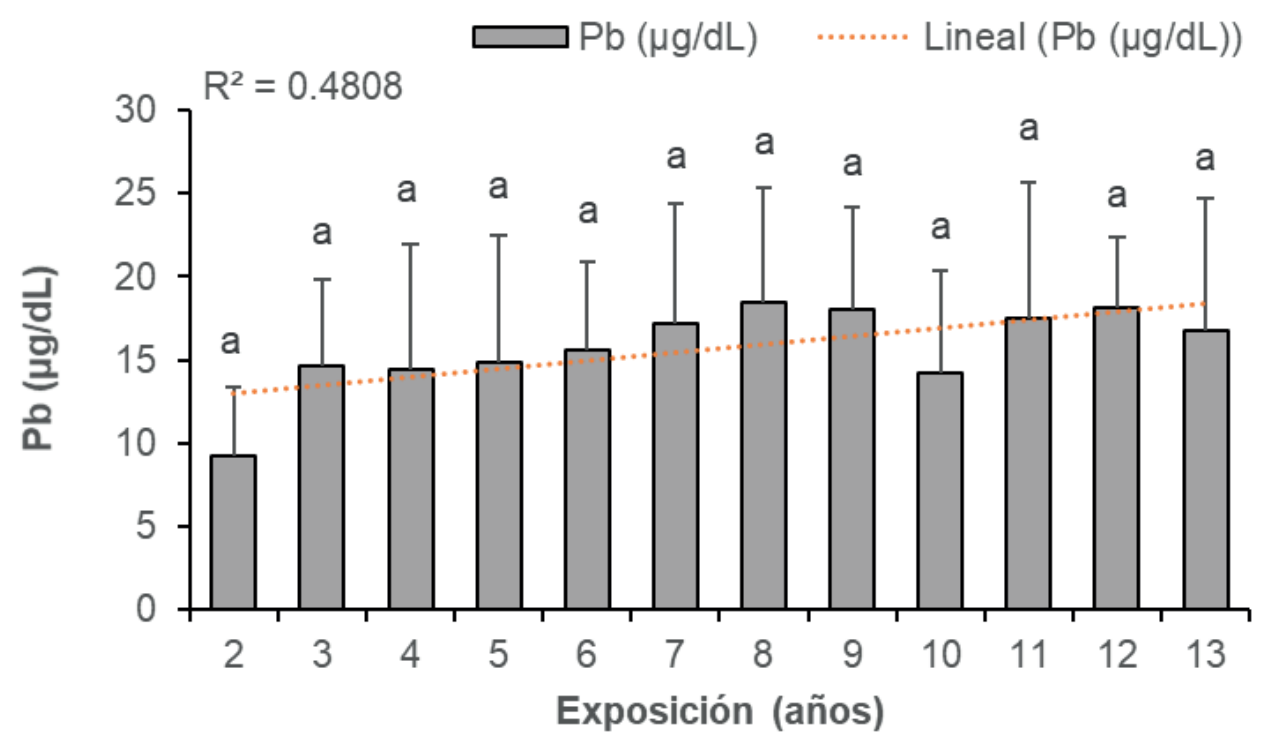

Nota: Letras iguales indican que no existen diferencias significativas según Anova de una vía y test a posteriori de Tukey (P > 0.05).

Figura 7. Análisis de componentes principales entre las variables concentración de plomo en sangre (Pb), sexo de los trabajadores, cargo, edad y años de exposición al Pb en los trabajadores de las fábricas de baterías

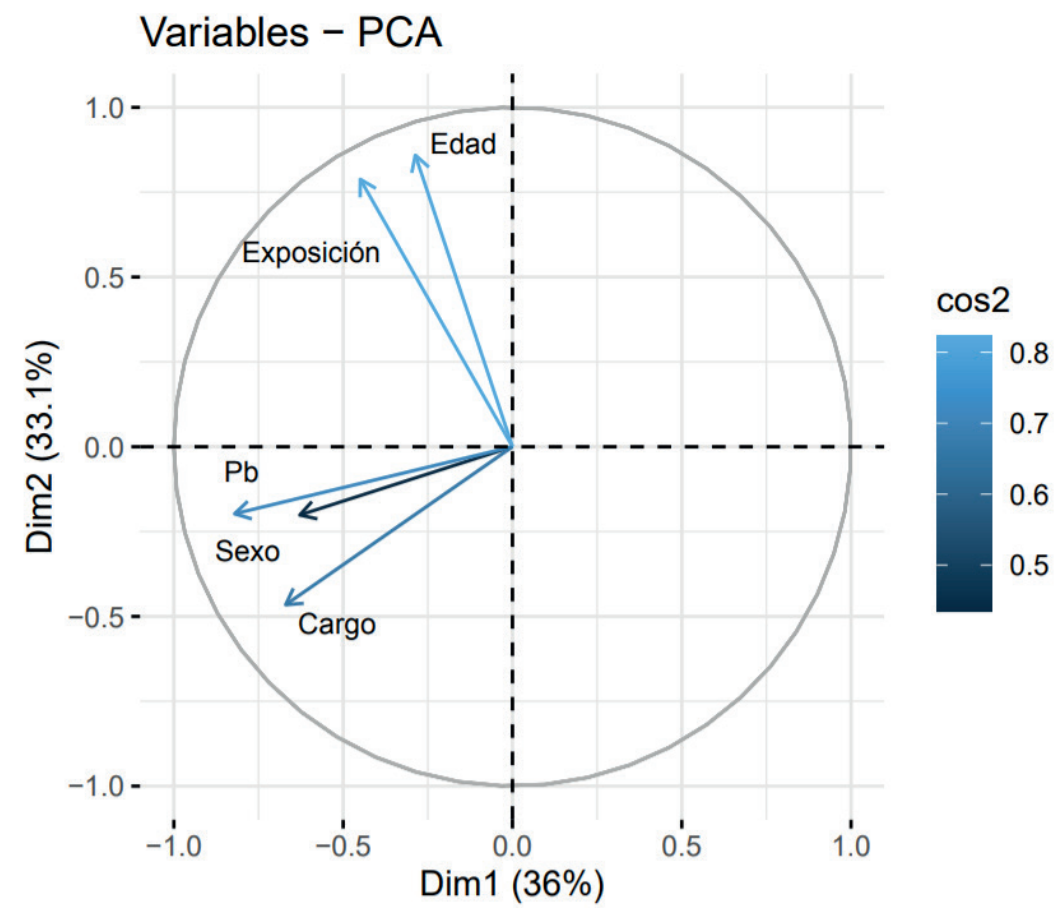


Con respecto a los riegos a los cuales están expuestos los trabajadores en estudio, en la Tabla 2 se muestra, según el rango de $\mathrm{Pb}$ en sangre, cuáles son los riesgos y posibles efectos para los empleados de las fábricas de baterías. Solo el $4.55 \%$ de los empleados no presentó riesgo por envenenamiento; un 13.64 \% mostró un riesgo bajo y la mayor parte de los empleados (78.79 \%) se encontraron en riesgo medio, solo en un $3.33 \%$ se observó alto riesgo, quienes podrían sufrir de cáncer, daños renales y anomalías en el esperma. Afortunadamente, no se observaron casos de $70 \mu \mathrm{g} / \mathrm{dL}$, por lo que se descarta la plumbemia en la población.

Tabla 2. Análisis de riesgo y posibles efectos sobre la salud de los trabajadores de las fábricas de baterías y el grupo control según la concentración de Pb en sangre

\begin{tabular}{|c|c|c|c|c|c|}
\hline $\begin{array}{l}\text { Rangos de } \\
\mathrm{Pb}(\mu \mathrm{g} / \mathrm{dL})\end{array}$ & $\begin{array}{c}\text { Norma } \\
\text { de referencia }\end{array}$ & Frecuencia & $\begin{array}{c}\text { Porcentaje } \\
(\%)\end{array}$ & $\begin{array}{l}\text { Análisis } \\
\text { de riesgo }\end{array}$ & $\begin{array}{l}\text { Posibles efectos } \\
\text { sobre la salud }\end{array}$ \\
\hline \multicolumn{6}{|c|}{ Trabajadores de las fábricas de baterías } \\
\hline Menos de 5 & $\begin{array}{l}\text { Valor de } \\
\text { referencia para } \\
\text { adultos según } \\
\text { la CDC }\end{array}$ & 9 & $4.55 \%$ & $\begin{array}{l}\text { No hay riesgo de } \\
\text { envenenamiento } \\
\text { por plomo }\end{array}$ & $\begin{array}{c}\text { Solo afectaría a mujeres } \\
\text { embarazadas con reducción } \\
\text { en el crecimiento de los fetos } \\
\text { si el valor supera } 2.5 \mu \mathrm{g} / \mathrm{dL} \\
\text { (Xie et al., 2013). }\end{array}$ \\
\hline De 6 a10 & $\begin{array}{l}\text { Límite máximo } \\
\text { permisible } \\
\text { según la OMS }\end{array}$ & 27 & $13.64 \%$ & $\begin{array}{l}\text { Riesgo bajo de } \\
\text { envenenamiento } \\
\text { por plomo }\end{array}$ & $\begin{array}{l}\text { Trastornos del estado de } \\
\text { ánimo, aumento de riesgo de } \\
\text { sufrir enfermedades cardio- } \\
\text { vasculares (ASTDR, 2020). }\end{array}$ \\
\hline De 11 a 30 & $\begin{array}{c}\text { Límite máximo } \\
\text { para exposición } \\
\text { laboral según } \\
\text { ACGIH }\end{array}$ & 156 & $78.79 \%$ & $\begin{array}{l}\text { Riego medio de } \\
\text { envenenamiento } \\
\text { por plomo }\end{array}$ & $\begin{array}{c}\text { Pérdida de audición, proble- } \\
\text { mas de equilibrio, daño en el } \\
\text { esperma, pérdida de funcio- } \\
\text { namiento neuronal, disminu- } \\
\text { ción de la función cognitiva, } \\
\text { anemia y daño renal (ASTDR, } \\
\text { 2020). }\end{array}$ \\
\hline De 30 a 40 & $\begin{array}{l}\text { Límite de } \\
\text { tolerancia en } \\
\text { personas adul- } \\
\text { tas expuesta } \\
\text { a Pb según las } \\
\text { normas OSHAS }\end{array}$ & 6 & $3.03 \%$ & $\begin{array}{l}\text { Riesgo alto de } \\
\text { envenenamiento } \\
\text { por plomo }\end{array}$ & $\begin{array}{l}\text { Aumento en el riesgo de } \\
\text { cáncer de riñón, sistema } \\
\text { nervioso, hígado, estómago } \\
\text { y esófago (Nersesyan et } \\
\text { al., 2016) y anomalías en el } \\
\text { esperma. }\end{array}$ \\
\hline \multicolumn{6}{|c|}{ Control } \\
\hline Menos de 5 & $\begin{array}{l}\text { Valor de } \\
\text { referencia para } \\
\text { adultos según } \\
\text { la CDC }\end{array}$ & 21 & $100 \%$ & $\begin{array}{l}\text { No hay riesgo de } \\
\text { envenenamiento } \\
\text { por plomo }\end{array}$ & No hay efectos \\
\hline
\end{tabular}

Los riesgos según el puesto de trabajo evidenciaron que solo el personal de montaje (10.64 \%) y el de formación de baterías (2.17 \%) presentan un alto riesgo de envenenamiento por plomo (Figura 8). Por el contrario, el único puesto que no presenta riesgo de intoxicación es el de oficina al igual que el grupo control y el de Recursos Humanos con riesgo bajo (50.00 \%) a nulo (50.00\%). 
Figura 8. Riesgos de envenenamiento por plomo en los trabajadores de las fábricas de baterías según el puesto de trabajo y en el grupo control

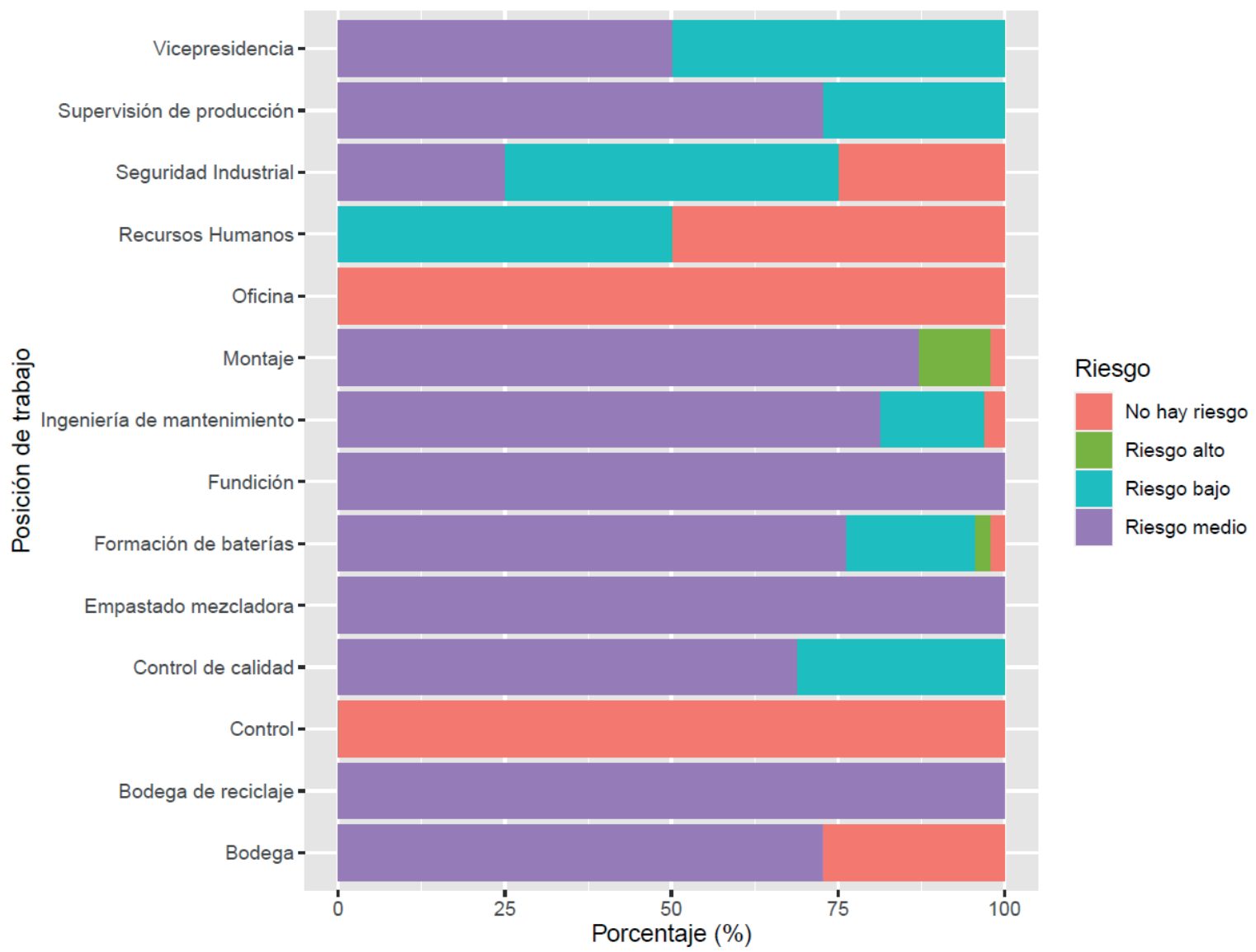

\section{Discusión}

Se determinaron los niveles de plomo en sangre en trabajadores de fábricas de baterías ubicadas en Guayaquil-Ecuador, donde se detectó el metal en el 100 \% de los empleados, con un rango de 3.50-38.00 $\mu \mathrm{g} / \mathrm{dL} \mathrm{Pb}$. Dentro de las causas de la contaminación por plomo en los trabajadores se encuentran la falta de higiene, problemas de ventilación en la empresa, no utilizar la ropa adecuada, la ingestión de alimentos en las áreas contaminadas y la falta de capacitación sobre los impactos del plomo en la salud. También se conoció que algunos trabajadores llevan la ropa usada durante la jornada laboral a sus hogares, lo que podría contaminar a sus familiares; asimismo, algunos empleados no utilizaban las máscaras porque les molestaban o les generaban calor. Las vías de ingreso del Pb en estos trabajadores fue la inhalación de partículas de plomo, la ingesta de alimentos en las zonas contaminadas y el contacto con la ropa impregnada con plomo.

Comparativamente, las concentraciones de $\mathrm{Pb}$ en los trabajadores de las fábricas de baterías de Guayaquil fueron semejantes a los resultados encontrados en Argentina $19.23 \mu \mathrm{g} /$ dL (Bilotta, Merodo, \& Aníbal, 2013), y menores a las reportadas en trabajadores de fábricas de Quito, donde se registraron niveles de $79.90 \mu \mathrm{g} / \mathrm{dL}(\mathrm{Cruz}, 2015)$ y a las reportadas en otros 
países, tales como Perú $37.70 \mu \mathrm{g} / \mathrm{dL}$ (Ramírez, 2008), Brasil $59.40 \mu \mathrm{g} / \mathrm{dL}$ (Gottesfeld \& Pokhrel, 2011), Venezuela $45.80 \mu \mathrm{g} / \mathrm{dL}$ (Gottesfeld \& Pokhrel, 2011), India 26.20-36.80 $\mu \mathrm{g} / \mathrm{dL}$ (Mishra et

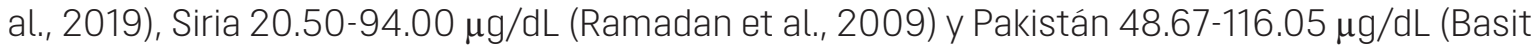
et al., 2015). Es importante mencionar que las fábricas de baterías analizadas en este trabajo cuentan con licencia ambiental en Ecuador y cumplen con las medidas de seguridad y salud ocupacional, lo que se refleja como menores niveles de plomo en su personal. Por el contrario, se han registrado altos niveles de $\mathrm{Pb}$ en trabajadores de fábricas informales de reciclado de baterías ubicadas en el sur del Perú, donde las concentraciones de Pb está entre 13.50 a $72.60 \mu \mathrm{g} / \mathrm{dL}$, debido a las precarias condiciones de higiene y seguridad ocupacional tienen estas fábricas (Ramírez, 2008).

En cuanto a la exposición ocupacional, la máxima concentración de los trabajadores del presente estudio fue de $38.30 \mu \mathrm{g} / \mathrm{dL}$ superando las concentraciones establecidas por la ACGIH, donde el valor límite en sangre es de $30 \mu \mathrm{g} / \mathrm{dL}$. Sin embargo, según la OSHAS sus valores de exposición permitida están entre $10 \mu \mathrm{g} / \mathrm{dL}$ a $40 \mu \mathrm{g} / \mathrm{dL}$.

Es preocupante que los límites laborales sean tan elevados tomando en cuenta que a partir de $10 \mu \mathrm{g} / \mathrm{dL}$ se considera que existe envenenamiento por plomo e incluso se ha demostrado que aun a menores concentraciones algunas personas pueden sufrir de aumento de la presión arterial, temblores y disfunción renal (Devóz et al., 2017), y después de los $30 \mu \mathrm{g} / \mathrm{dL}$ aumenta el riesgo de padecer de cáncer (Nersesyan et al., 2016). Además, Instituto de Sanimetría y Evaluación Sanitaria reporta que la contaminación por Pb causó 1,06 millones de defunciones en el año 2017 y estimó que este metal pesado ocasionó el 10.30 \% de las cardiopatías hipertensivas, el $6.20 \%$ de los accidentes cerebrovasculares y el $5.60 \%$ de las cardiopatías isquémicas a nivel mundial (OMS, 2019). Es por ello que se propone disminuir los límites permisibles para los trabajadores en Ecuador, mediante una norma que dicte que los trabajadores no superen los $25 \mu \mathrm{g} / \mathrm{dL}$ de plomo.

En lo referente al puesto de trabajo, se demostró que el riesgo de contaminación es mayor en las áreas de montaje, formación de batería y fundición de plomo, probablemente porque se trabaja directamente con el plomo y este se une al material particulado y es inhalado por los trabajadores; y por el contrario, en las zonas de oficinas, recursos humanos y seguridad industrial, los niveles de plomo de los trabajadores no superaron los límites máximos permisibles de la OMS, por no estar en contacto directo con las áreas de trabajo contaminadas. Al igual que en esta investigación se ha descrito que el proceso de fundición del plomo genera un riesgo importante de intoxicación ocupacional por plomo y afecta tanto al trabajador como a su familia (Ramírez, 2008); de igual manera, en otras fábricas de baterías, los niveles de plomo en sangre son significativamente mayores en las secciones de pulido, corte y montaje en comparación con la sección de administración (Ravichandran et al., 2005). Asimismo, Ramadan et al. (2009) encontraron diferencias en el contenido de plomo en sangre en los diferentes sitios de trabajo en una fábrica de batería.

El sexo también fue determinante en los niveles de plomo porque muestra una tendencia a presentar mayores cantidades de plomo los hombres tanto en el grupo control como en los trabajadores de las fábricas de baterías. La explicación en la fábrica es que los hombres son los que trabajan en las áreas más contaminadas, mientras que las mujeres trabajan en las áreas de oficina donde la exposición al Pb es muy baja. En el grupo control podría deberse a otra fuente de contaminación como la contaminación atmosférica y a la ingesta de alimentos contaminados. 
En líneas generales, los hombres ingieren mayor cantidad de alimentos que las mujeres y por ello podrían acumular mayores concentraciones del metal pesado.

Respecto al grupo control, el 62.00 \% presentó plomo en sangre a pesar de que esta concentración está por debajo del límite establecido por la OMS, y, al no estar expuestos ocupacionalmente al $\mathrm{Pb}$, se comprueba que existen otras vías alternas de contaminación. Una de las fuentes de contaminación podría ser de origen atmosférico por el polvo que se genera en las calles de Guayaquil. En este sentido, se ha descrito que el polvo es uno de los portadores más importantes de metales pesados (Padoan et al., 2017) y se ha demostrado que está constituido por tierra, materiales de construcción depositados, partículas en el aire, hollín y humos producidos por las industrias y los vehículos (Hou et al., 2019). Satián (2019), en su estudio realizado en Guayaquil, mostró la contaminación por plomo en el hollín procedente de la Metrovía (131.00 mg/kg y 127.00 mg/kg), el que puede ser inhalado como material particulado.

Del mismo modo, un estudio hecho en México por Morán (2012) demostró la concentración de plomo en sangre en personas no expuestas ocupacionalmente, donde la mayor concentración fue de $9.21 \mu \mathrm{g} / \mathrm{dL}$, superior a la presente en el grupo control de Guayaquil. Por esta razón, se puede afirmar que sí existen otras fuentes de contaminación como la contaminación atmosférica. Otra vía de exposición a este metal pesado es a través de los alimentos. Un estudio realizado por Pernía et al. (2015) demostró que la leche en polvo puede contener hasta $7.77 \mathrm{mg} /$ kg de Pb. También se ha encontrado plomo en el arroz, soya, cacao y café, alimentos de alto consumo en Ecuador, pueden representar una fuente importante de plomo (Benavides et al., 2021), así como en el agua potable de la ciudad de Guayaquil (Cipriani-Ávila et al., 2020).

Los valores hallados en el grupo control son bajos en comparación a los valores mundiales registrados en los años ochenta (mayor a $10 \mu \mathrm{g} / \mathrm{dL}$ ), gracias a los esfuerzos para eliminar el plomo de la gasolina, la pintura y el agua potable, lo que se ha reflejado como una reducción sustancial en los niveles de plomo en la sangre humana (Hwang et al., 2019).

Para futuros estudios se recomienda analizar, además, del contenido de Pb en sangre algunos bioindicadores del daño generado por el metal pesado, tales como las coproporfirinas, ácido aminolevulínico en la orina, pirimidina-5-nucleotidasa, protoporfirina y actividad deshidratasa del ácido $\delta$-aminolevulínico (ALAD) en eritrocitos (Sudjaroen \& Suwannahong, 2017). También se recomienda incrementar el número de muestras en el grupo control para obtener un valor representativo de la población guayaquileña.

\section{Conclusiones y recomendaciones}

Esta investigación encontró concentraciones de $\mathrm{Pb}$ en sangre en un rango de $3.50 \mu \mathrm{g} / \mathrm{dL}$ a $38.30 \mu \mathrm{g} / \mathrm{dL}$ en los trabajadores de fábricas de baterías de Guayaquil. En cuanto a la comparación de Pb en sangre frente a la normativa internacional, se demostró que el 81.82 \% de los trabajadores exceden el límite máximo establecido por la OMS, y el 3.03 \% exceden el valor de la ACGIH, sin embargo, en relación con la normativa OSHAS se encuentran por debajo del límite.

Asimismo, se determinaron los valores bases de $\mathrm{Pb}$ en sangre de los voluntarios no expuestos a este metal pesado en la ciudad de Guayaquil, que variaron de 0.00 a $2.20 \mu \mathrm{g} / \mathrm{dL}$ dando como valor promedio $0.68 \mu \mathrm{g} / \mathrm{dL}$. Además, se confirmó la hipótesis, debido a que las concentraciones de $\mathrm{Pb}$ en sangre de los trabajadores fueron significativamente mayores con respecto a las personas no expuestas ocupacionalmente a este metal pesado, lo que indica un riesgo ocupacional para las personas que laboran en esta actividad. 
Comparativamente, los hombres presentaron mayores concentraciones de $\mathrm{Pb}$ que las mujeres; aparte, se constató que los puestos de trabajo donde existe un alto riesgo de envenenamiento por plomo fueron el área de montaje y formación de baterías. Por el contrario, no se encontró correlación entre la edad y la concentración de plomo en sangre de los trabajadores de baterías ni en la población control y una correlación moderada entre los niveles de Pb y los años de exposición al metal.

Con la finalidad de proteger la salud de los trabajadores de fábricas de baterías, se enumeran a continuación unas recomendaciones para mitigar los riesgos a exposición al Pb:

1. Aislar los procesos para evitar que los gases volátiles afecten al personal, y, de manera continua, revisar y cambiar los filtros de aire.

2. En toda el área industrial es necesario usar extractores de aire para eliminar el material particulado que contiene Pb y causa problemas de salud en los trabajadores.

3. Capacitar al personal sobre los peligros del envenenamiento por plomo y el uso apropiado de los equipos de protección.

4. El personal debe utilizar ropa adecuada: camisas con mangas largas, pantalón largo y botas de seguridad.

5. El personal debe utilizar equipos de protección personal, tales como guantes, lentes de seguridad, mascarillas de doble filtro. Se recomienda la 3m 6200 filtro 7093, que deberá ser cambiado cada 2 meses. En el caso de no utilizar las mascarillas, se debe amonestar al personal. Además, todos los equipos deben ser lavados, cambiados regularmente y deben guardarse en compartimientos separados de la ropa limpia.

6. Se recomienda que todo el personal consuma diariamente zinc, hierro, magnesio y vitamina $\mathrm{C}$ para disminuir la absorción del $\mathrm{Pb}$ por competencia.

7. Rotar al personal de sus puestos de trabajo cuando los trabajadores muestren plomo en la sangre en una concentración superior a $25 \mu \mathrm{g} / \mathrm{dL}$.

8. Vigilar que el personal no ingiera alimentos en el área de procesos.

9. A la hora de la comida, el personal debe cumplir con las medidas de higiene, tales como lavado de manos y cambio de uniforme.

10. No fumar en el trabajo. También debe prohibirse la tenencia del tabaco en el lugar de trabajo porque este puede contaminarse. Aunque se fume luego, constituye una vía de exposición potencial.

11. Debe ser obligatorio el uso de las duchas diariamente, al término de la jornada, con el fin de evitar que este agente químico se deposite en la piel, uñas y cabello del trabajador.

12. Al término de la jornada, la ropa del trabajador se debe lavar dentro de las instalaciones. El trabajador no podrá llevarse el uniforme para su hogar para evitar que sus familiares se contaminen con $\mathrm{Pb}$.

13. Instalar duchas y lavaojos en los sectores donde se trabaje con plomo y ácido sulfúrico.

14. Realizar monitoreos de aire de forma bimensual para salvaguardar a los trabajadores y que el área de trabajo esté por debajo de los límites establecidos en aire $<50 \mu \mathrm{g} / \mathrm{m}^{3}$. 


\section{Referencias}

Ahn, J., Park, M. Y., Kang, M. Y., Shin, I. S., An, S., \& Kim, H. R. (2020). Occupational Lead Exposure and Brain Tumors: Systematic Review and Meta-analysis. International Journal of Environmental Research and Public Health, 17(11), 1-14. https://doi.org/10.3390/ijerph17113975

Alatise, O. I., \& Schrauzer, G. N. (2010). Lead Exposure: A Contributing Cause of The Current Breast Cancer Epidemic in Nigerian Women. Biological Trace Element Research, 136(2), 127-139. https://doi. org/10.1007/s12011-010-8608-2

Agency for Toxic Substances and Disease Registry. (2020). Toxicological Profile for Lead. https://www. atsdr.cdc.gov/toxprofiles/tp13.pdf

Awadalla, A., Mortada, W. I., Abol-Enein, H., \& Shokeir, A. A. (2020). Correlation between Blood Levels of Cadmium and Lead and The Expression of microRNA-21 in Egyptian Bladder Cancer Patients. Heliyon, 6(12), e05642. https://doi.org/10.1016/j.heliyon.2020.e05642

Ballantyne, A. D., Hallett, J. P., Jason, D., Shah, N., Payne, D. J., \& Payne, D. J. (2018). Lead Acid Battery Recycling for The Twenty-first Century. R. Soc. Open Sci., 5, 171368. https://doi.org/10.1098/ rsos. 171368

Baloch, S., Kazi, T. G., Baig, J. A., Afridi, H. I., \& Arain, M. B. (2020). Occupational Exposure of Lead and Cadmium on Adolescent and Adult Workers of Battery Recycling and Welding Workshops: Adverse Impact on Health. Science of the Total Environment, 137549. https://doi.org/10.1016/j.scitotenv.2020.137549

Barry, V., \& Steenland, K. (2019). Lead Exposure and Mortality Among U.S. Workers in A Surveillance Program: Results from 10 Additional Years of Follow-up. Environmental Research, 177, 108625. https://doi.org/10.1016/j.envres.2019.108625

Basit, S., Karim, N., \& Munshi, A. B. (2015). Occupational Lead Toxicity in Battery Workers. Pakistan Journal of Medical Sciences, 37(4), 775-780. https://doi.org/10.12669/pjms.314.7066

Benavides, A., Romero, B., Pérez, I., Pernía, B. (2021). Contaminación por metales pesados en alimentos de origen vegetal en Ecuador: Una propuesta para su mitigación. Granja, In Press.

Bilotta, M. C., Merodo, P., \& Godoy Ortiz, A. (2013). Exposición a la contaminación con plomo en taller de ensamble de baterías. Ciencia \& Trabajo, 15(48), 158-164. https://doi.org/10.4067/s071824492013000300009

Cevallos, F. (2013). Evaluación y correlación clínica de los niveles de plumbemia, en los trabajadores que elaboran acumuladores eléctricos, para la implementación de medidas de control. [Tesis de licenciatura, Universidad Internacional SEK, Quito, Ecuador. Repositorio Universidad Internacional SEK Ecuador. https://bit.ly/38VX3eD

Chen, X., Zhu, G., Wang, Z., Zhou, H., He, P., Liu, Y., \& Jin, T. (2019). Ecotoxicology and Environmental Safety The association between Lead and Cadmium Co-exposure and Renal Dysfunction. Ecotoxicology and Environmental Safety, 173, 429-435. https://doi.org/10.1016/j.ecoenv.2019.01.121

Chowdhury, R., Ebelt Sarnat, S., Darrow, L., Mcclellan, W., \& Steenland, K. (2014). Mortality among Participants in A Lead Surveillance Program. Environmental Research, 132, 100-104. https://doi. org/10.1016/j.envres.2014.03.008

Cipriani-Avila, I., Molinero, J., Jara-Negrete, E., Barrado, M., Arcos, C., Mafla, S., Custode, F., Vilaña, G., Carpintero, N., \& Ochoa-Herrera, V. (2020). Heavy metal assessment in drinking waters of Ecuador: Quito, Ibarra and Guayaquil. Journal of Water and Health, 18(6), 1050-1064. https://doi. org/10.2166/wh.2020.093

Dean, J. R., Amaibi, P. M., Okorie, A., \& Entwistle, J. A. (2020). A Critical Evaluation of The Use and 'Misuse' of As and Pb Bioaccessibility Data in Human Health Risk Assessments. Environmental Research, 189, 109915. https://doi.org/10.1016/j.envres.2020.109915

Devóz, P. P., Gomes, W. R., Araújo, M. L. De, Ribeiro, L., Pedron, T., Maria, L., Antunes, G., Lemos, B., Jr, F. B., Rafael, G., Barcelos, M., Ribeiro, D. L., Pedron, T., Maria, L., Antunes, G., Batista, B. L., Jr, F. B., 
Rafael, G., Barcelos, M., \& Pb, L. (2017). Lead (Pb) Exposure Induces Disturbances in Epigenetic Status in Workers Exposed To This Metal. Journal of Toxicology and Environmental Health, Part A, 80(19-21), 1098-1105. https://doi.org/10.1080/15287394.2017.1357364

Fry, K. L., Wheeler, C. A., Gillings, M. M., Flegal, A. R., \& Taylor, P. (2020). Anthropogenic Contamination of Residential Environments from Smelter As, Cu and Pb Emissions: Implications for Human Health. Environmental Pollution, 262, 114235. https://doi.org/10.1016/j.envpol.2020.114235

Gaza, M. A., Hakim, L., Sabarudin, A., \& Bambang, S. (2017). Evaluation on Mercury, Cadmium, and Lead in the Hair Sample as an Indicator of Autism for Children. 9(12), 710-715. https://bit.ly/3bOEQS6

Gottesfeld, P., \& Pokhrel, A. K. (2011). Review: Lead Exposure in Battery Manufacturing and Recycling in Developing Countries and Among Children in Nearby Communities. Journal of Occupational and Environmental Hygiene, 8(9), 520-532. https://doi.org/10.1080/15459624.2011.601710

Himani, Kumar, R., Ansari, J. A., Mahdi, A. A., Sharma, D., Karunanand, B., \& Datta, S. K. (2020). Blood Lead Levels in Occupationally Exposed Workers Involved in Battery Factories of Delhi-NCR Region: Effect on Vitamin D and Calcium Metabolism. Indian Journal of Clinical Biochemistry, 35(1), 8087. https://doi.org/10.1007/s12291-018-0797-z

Hou, S., Zheng, N., Tang, L., Ji, X., Li, Y., \& Hua, X. (2019). Pollution Characteristics, Sources, and Health Risk Assessment of Human Exposure to $\mathrm{Cu}, \mathrm{Zn}, \mathrm{Cd}$ and $\mathrm{Pb}$ Pollution in Urban Street Dust Across China between 2009 and 2018. Environment International, 128(julio), 430-437. https://doi. org/10.1016/j.envint.2019.04.046

Hwang, Y., Kate, C., \& Lin, P. (2019). Globally Temporal Transitions of Blood Lead Levels of Preschool Children across Countries of Different Categories of Human Development Index. Science of the Total Environment, 659(17), 1395-1402. https://doi.org/10.1016/j.scitotenv.2018.12.436

Instituto Nacional de Estadísticas y Censos. (2015). Compendio estadístico 2016. https://www.ecuadorencifras.gob.ec/compendio-estadistico-2016/

Johansson, E., Yahia, M. W., Arroyo, I., \& Bengs, C. (2018). Outdoor Thermal Comfort in Public Space in Warm-humid Guayaquil, Ecuador. International Journal of Biometeorology, 62(3), 387-399. https://doi.org/10.1007/s00484-017-1329-x

Kalahasthi, R., Bagepally, B. S., \& Tapu, B. (2019). Association of Musculoskeletal Disorders and Inflammation Markers in Workers Exposed to Lead ( Pb ) from Pb - Battery Manufacturing Plant. Indian Journal of Occupational and Environmental Medicine, 23(2), 68-72. https://doi.org/10.4103/ ijoem.IJOEM_192_18

Meng, Y., Tang, C., Yu, J., Meng, S., \& Zhang, W. (2020). Exposure to Lead Increases The Risk of Meningioma and Brain Cancer: A Meta-analysis. Journal of Trace Elements in Medicine and Biology, 60(December 2019), 126474. https://doi.org/10.1016/j.jtemb.2020.126474

Mishra, S., Kannan, S., Manager, C., Statistics, A., Comments, R., \& Alert, E. (2019). Association of Musculoskeletal Disorders and Inflammation Markers in Workers Exposed to Lead $(\mathrm{Pb})$ from Pb-battery Manufacturing plant. Indian Journal of Occupational and International Medicine, 23(1), 8-13. https://doi.org/10.4103/ijoem.IJOEM_192_18

Morán, J. (2012). La contaminación ambiental y ocupacional por plomo y sus efectos en la salud reproductiva masculina, evidencia de daño al ADN. Revista Iberoamericana de Las Ciencias de La Salud, 1(2), 1-36. https://dialnet.unirioja.es/servlet/articulo?codigo $=5045746$

Nersesyan, A., Kundi, M., Waldherr, M., Setayesh, T., Misik, M., Wultsch, G., Filipic, G., \& Siegfried, B. (2016). Results of Micronucleus Assays with Individuals Who Are Occupationally and Environmentally Exposed To Mercury, Lead And Cadmium. Mutation Research-Reviews in Mutation Research. https://doi.org/10.1016/j.mrrev.2016.04.002

Nouioui, M. A., Araoud, M., Milliand, M., \& Bessueille-barbier, F. (2019). Biomonitoring Chronic Lead Exposure among Battery Manufacturing Workers in Tunisia. Environmental Science and Pollution Research, 26(8), 7980-7993. https://doi.org/10.1007/s11356-019-04209-y 
Organización Mundial de la Salud. (2019). Intoxicación por plomo y salud. https://www.who.int/es/news$\mathrm{room} /$ fact-sheets/detail/lead-poisoning-and-health

Padoan, E., Romè, C., \& Ajmone-Marsan, F. (2017). Bioaccessibility and Size Distribution of Metals in Road Dust and Roadside Soils along A Peri-Urban Transect. Science of the Total Environment, 601602, 89-98. https://doi.org/10.1016/j.scitotenv.2017.05.180

Pernía, B., Mero, M., Bravo, K., Ramírez, N., López, D., Muñoz, J., \& Egas, F. (2015). Detección de cadmio y plomo en leche de vaca comercializada en la ciudad de Guayaquil, Ecuador. Rev. Cient. Cien. Nat. Ambien., 8(2), 81-86. https://bit.ly/38YWDUT

Ramadan, A. A., Mandil, H., \& Ismail, M. H. (2009). Determination of Lead Pollutants in Acid Batteries and Liquid Gases in Aleppo-Syrian Company Using Total Suspended Particulate and Chemical Trap Methods. Asian Journal of Chemistry, 21(8), 6562-6568. https://bit.ly/3iqN6Jn

Ramírez, A. (2008). Exposición a plomo en trabajadores de fábricas informales de baterías: Workers' Lead Exposure in Informal Batteries Factories. An Fac Med., 69(2), 104-107. https://bit.ly/3isPteq

Ravichandran, B., Ravibabu, K., Raghavan, S., Krishnamurthy, V., Rajan, B. K., \& Rajmohan, H. R. (2005). Battery Manufacturing Unit in India. Journal of Occupational Health, 47(4), 350-353. https:// doi.org/10.1539/joh.47.350

Rousseau, M. C., Parent, M. E., Nadon, L., Latreille, B., \& Siemiatycki, J. (2007). Occupational Exposure To Lead Compounds and Risk of Cancer Among Men: A Population-based Case-control Study. American Journal of Epidemiology, 166(9), 1005-1014. https://doi.org/10.1093/aje/kwm183

Steenland, K., \& Boffetta, P. (2000). Lead and Cancer in Humans: Where Are We Now? American Journal of Industrial Medicine, 38(3), 295-299. https://doi.org/10.1002/10970274(200009)38:3<295::AID-AJIM8>3.0.CO;2-L

Steenland, K., Barry, V., Anttila, A., Sallmen, M., Mueller, W., Ritchie, P., McElvenny, D. M., \& Straif, K. (2019). Cancer Incidence Among Workers With Blood Lead Measurements in Two Countries. Occupational and Environmental Medicine, 76(9), 603-610. https://doi.org/10.1136/oemed-2019-105786

Stojsavljevi, A., Borkovi, S., Vujoti, L., Gruji, D., Gavrovi, M., \& Manojlovi, D. (2019). Ecotoxicology and Environmental Safety. The Human Biomonitoring Study in Serbia: Background Levels For Arsenic, Cadmium, Lead, Thorium And Uranium In The Whole Blood Of Adult Serbian Population. Ecotoxicology and Environmental Safety, 169, 402-409. https://doi.org/10.1016/j.ecoenv.2018.11.043

Sudjaroen, Y., \& Suwannahong, K. (2017). Biomarker Related Lead Exposure of Industrial Battery's Workers. Annals of Tropical Medicine and Public Health, 10(1), 194-198. https://doi.org/10.4103/17556783.196523

Téllez-Rojo, M. M., Bautista-Arredondo, L., Leng, L., Richardson, V., Estrada-Sánchez, D., Ávila-Jiménez, L., Ríos, C., Cantoral-Preciado, A., Romero-Martínez, M., Flores-Pimentel, D., Melo-Zurita, M. C., Romero-Ramírez, A., León-Mazón, M. A., Montes, S., Fuller, R., \& Hernández-Ávila, M. (2017). Intoxicación por plomo y nivel de marginación en recién nacidos de Morelos, México. Salud Pública de México, 59(3), 218-226. https://doi.org/10.21149/8045

Torres Del salto, R., Constante, M., Torres, B., \& Rendón, I. (2017). Evaluación climática para determinar las estrategias bioclimáticas de una vivienda dúplex en la ciudad de Durán. Dominio de Las Ciencias, 3(3), 865-883. https://dialnet.unirioja.es/servlet/articulo?codigo=6244038

Xie, X., Ding, G., Cui, C., Chen, L., Gao, Y., Zhou, Y., Shi, R., \& Tian, Y. (2013). The Effects of Low-Level Prenatal Lead Exposure on Birth Outcomes. Environmental Pollution, 175, 30-34. https://doi. org/10.1016/j.envpol.2012.12.013

Yabe, J., Nakayama, S. M. M., Ikenaka, Y., Yohannes, Y. B., Bortey-sam, N., Nketani, A., Ntapisha, J., Mizukawa, H., Umemura, T., \& Ishizuka, M. (2018). Chemosphere Lead and Cadmium Excretion in Feces and Urine of Children from Polluted Townships Near A Lead-Zinc Mine in Kabwe, Zambia. Chemosphere, 202, 48-55. https://doi.org/10.1016/j.chemosphere.2018.03.079 